\title{
Do the Paths of Sequential Vein Grafts Influence the Outcomes of Coronary Artery Bypass Surgeries?
}

\author{
Yuan-Hsi Tseng, ${ }^{1}$ Chih-Chen Kao, ${ }^{1}$ Chien-Chao Lin, ${ }^{1}$ Chien-Wei Chen, ${ }^{2}$ Ming-Shian Lu, ${ }^{1}$ \\ Chu-Hsueh Lu, ${ }^{1}$ Yao-Kuang Huang ${ }^{1}$ \\ ${ }^{1}$ Division of Thoracic and Cardiovascular Surgery, Chia Yi Chang Gung Memorial Hospital, Chia-Yi and Chang Gung University, \\ College of Medicine, Taoyuan, Taiwan; ${ }^{2}$ Department of Diagnostic Radiology, Chang Gung Memorial Hospital, Chang-Gung \\ University, Chiayi and Taoyuan, Taiwan
}

\section{ABSTRACT}

Background: The use of a sequential vein graft (SVG) in coronary artery bypass grafting (CABG) in multi-vessel coronary disease is common. This study aimed to investigate the influence of the paths of SVGs on the outcomes of CABG.

Methods: From January 2011 to June 2017, 126 patients underwent elective isolated CABG. If the path of the SVG was from the aorta to the right coronary artery (RCA)/ posterior descending artery (PDA) to the left circumflex artery (LCX)/ obtuse marginal artery $(\mathrm{OM})$, the patients were included in Group R. If the path was from the aorta to the LCX/OM to the RCA/PDA, the patients were included in Group L. The in-hospital and follow-up outcomes were analyzed.

Results: Group R had 69 patients, and Group L had 57 patients. Univariate analysis showed that Group L had a higher number of grafts $(P<.001)$ and less aortic crossclamping time $(P<.001)$ and total bypass time $(P=.001)$. Otherwise, Group L had 14 patients (19.3\%), who received first diagonal branch (D1) bypass grafting, while Group $\mathrm{R}$ had none $(P<.001)$. In the multivariate analysis, inhospital mortality from heart failure, postoperative acute kidney injury, medium-term mortality, and readmission for cardiac incidents were not associated with the SVG path.

Conclusion: The SVG path from the aorta to the LCX/ $\mathrm{OM}$ to the RCA/PDA facilitated the additional D1 bypass grafting, but the outcomes for this approach were not significantly different from those for the other path.

\section{INTRODUCTION}

Aside from left anterior descending artery (LAD) bypass grafting, the use of the great saphenous vein (GSV) for the bypass grafting of the other coronary branches is a common method of treatment for multi-vessel coronary disease. The means by which GSV grafts are anastomosed to the coronary

Received September 2, 2019; accepted October 23, 2019.

Correspondence: Yao-Kuang Huang, MD, PhD, Division of Thoracic and Cardiovascular Surgery, Chia-Yi Chang Gung Memorial Hospital, 6 West Section, Chia-Pu Rd, Putzu City, Chiayi County, Taiwan; +88605-3281200, ext. 2864 (e-mail: huang137@icloud.com). arteries include the use of individual vein grafts and sequential vein grafts (SVGs), and previous studies have proven that SVGs result in non-inferior outcomes and better blood flow than individual vein grafts [An 2018; Christenson 1997; Xiao

Table 1. Baseline characteristics and preoperative clinical conditions

\begin{tabular}{|c|c|c|c|}
\hline & $\begin{array}{l}\text { Group R } \\
(\mathrm{N}=69)\end{array}$ & $\begin{array}{l}\text { Group L } \\
(\mathrm{N}=57)\end{array}$ & $P$ \\
\hline Age (years) & $\begin{array}{c}63.0 \\
(58.0-72.0)\end{array}$ & $\begin{array}{c}64.0 \\
(56.0-73.0)\end{array}$ & .97 \\
\hline Sex & & & .81 \\
\hline Female, N (\%) & $12(17.4)$ & $9(15.8)$ & \\
\hline Male, N (\%) & $57(82.6)$ & $48(84.2)$ & \\
\hline \multicolumn{4}{|l|}{ Comorbidity } \\
\hline Diabetes, N (\%) & $45(65.2)$ & $38(66.7)$ & .86 \\
\hline Hyperlipidemia, N (\%) & $33(47.8)$ & $30(52.6)$ & .59 \\
\hline Hypertension, N (\%) & $56(81.2)$ & $43(75.4)$ & .44 \\
\hline $\mathrm{CrCl}^{*}(\mathrm{~mL} / \mathrm{min})$ & $\begin{array}{c}60.8 \\
(35.8-81.6)\end{array}$ & $\begin{array}{c}63.0 \\
(43.0-77.5)\end{array}$ & .87 \\
\hline PAOD, N (\%) & $6(8.7)$ & $4(7.0)$ & 1.00 \\
\hline History of stroke, N (\%) & $14(20.3)$ & $6(10.5)$ & .14 \\
\hline Chronic lung disease, N (\%) & $4(5.8)$ & $2(3.5)$ & .69 \\
\hline \multicolumn{4}{|l|}{ Clinical status } \\
\hline Recent MI, N (\%) & $36(52.2)$ & $34(59.6)$ & .40 \\
\hline Angina at rest, $\mathrm{N}(\%)$ & $41(59.4)$ & $35(61.4)$ & .82 \\
\hline Intubation before CABG, N (\%) & $4(5.8)$ & $3(5.3)$ & 1.00 \\
\hline IABP before CABG, N (\%) & $3(4.3)$ & $1(1.8)$ & .63 \\
\hline $\begin{array}{l}\text { EuroSCORE II predicted } \\
\text { mortality (\%) }\end{array}$ & $3.2(1.4-5.3)$ & $2.3(1.6-4.5)$ & .32 \\
\hline
\end{tabular}

*calculated with Cockcroft-Gault equation

CABG, coronary artery bypass grafting; $\mathrm{CrCl}$, creatinine clearance; EuroSCORE, European System for Cardiac Operative Risk Evaluation; IABP, intraaortic balloon pumping; MI, myocardial infarction; PAOD, peripheral arterial occlusive disease 
Table 2. Pre-operative examination findings

\begin{tabular}{lccc}
\hline & Group R & Group L & $P$ \\
\hline Pre-operative EF & $59(45.0-74.0)$ & $59.5(47.8-71.0)$ & 1.00 \\
EF > 50\%, N (\%)* & $43(62.3)$ & $39(68.4)$ & .43 \\
Severe coronary stenosis & & & \\
LM, N (\%) & $26(40.0)$ & $25(47.2)$ & .43 \\
LAD, N (\%) & $52(75.2)$ & $46(80.7)$ & .47 \\
LCX, N (\%) & $48(69.6)$ & $40(70.2)$ & .94 \\
RCA, N (\%) & $48(69.6)$ & $40(70.2)$ & .94 \\
SYNTAX score & $32.0(26.8-43.5)$ & $34.0(22.1-39.1)$ & .58 \\
\hline
\end{tabular}

*Two missing data

$\dagger$ The definition of significant stenosis was a finding of $\geq 50 \%$ for the LM and of $\geq 70 \%$ to other coronary branches

$E F$, ejection fraction; $L A D$, left anterior descending artery, LCX, left circumflex artery; LM, left main coronary artery; RCA, right coronary artery; SYNTAX, Synergy between PCl with Taxus and Cardiac Surgery

Table 3. Surgical data

\begin{tabular}{lccc}
\hline & Group R & Group L & $P$ \\
\hline Number of grafts & $3.0(3.0-3.0)$ & $3.0(3.0-3.5)$ & $<.001$ \\
GSV quality & & & .73 \\
Good, N (\%) & $55(79.7)$ & $45(78.9)$ & \\
Poor, N (\%) & $6(8.7)$ & $7(12.3)$ & \\
Small but elastic, N (\%) & $8(11.6)$ & $5(8.8)$ & \\
Ramus bypass grafting, N (\%) & 0 & $3(5.3)$ & .09 \\
D1 bypass grafting, N (\%) & 0 & $11(19.3)$ & $<.001$ \\
TBT & 130.0 & 111.0 & .001 \\
& $(113.0-151.0)$ & $(90.5-137.5)$ & \\
ACT & 99.0 & 77.0 & $<.001$ \\
Cardioversion, N (\%) & $(89.0-114.5)$ & $(62.0-94.5)$ & \\
Inotropic agent or IABP for & $11(15.9)$ & $10(17.5)$ & .81 \\
weaning off CPB, N (\%) & $16(23.2)$ & $12(21.1)$ & .77 \\
\hline
\end{tabular}

$\mathrm{ACT}$, aortic cross-clamping time; CPB, cardiopulmonary bypass; D1, first diagonal branch; GSV, great saphenous vein; IABP, intraaortic balloon pumping; TBT, total bypass time

2015; Kim 2011]. Other advantages of SVGs include a shorter required vein graft length, less aortic manipulation, the revascularization of more coronary arteries, and decreased likelihood of myocardial infarction or death when suffering proximal occlusion [Christenson 1998].

According to our past experiences, an SVG generally can take one of the following two paths: from the aorta to the right coronary artery (RCA) or posterior descending
Table 4. Postoperative outcomes and complications

\begin{tabular}{|c|c|c|c|}
\hline & Group R & Group L & $P$ \\
\hline $\begin{array}{l}\text { In-hospital mortality from } \\
\text { HF, N (\%) }\end{array}$ & $3(4.3)$ & $2(3.5)$ & 1.00 \\
\hline Duration of MV & $1.0(1.0-3.0)$ & $1.0(1.0-3.5)$ & .63 \\
\hline ICU stay & $4.0(4.0-6.0)$ & $4.0(3.0-6.0)$ & .09 \\
\hline Hospital stay & $15.0(11.0-20.0)$ & $11.0(8.5-20.0)$ & .15 \\
\hline \multicolumn{4}{|l|}{ Complications } \\
\hline New stroke, N (\%) & $3(4.3)$ & $3(5.3)$ & 1.00 \\
\hline Myocardial failure, N (\%) & $4(5.8)$ & $4(7.0)$ & 1.00 \\
\hline Respiratory failure, N (\%) & $5(7.2)$ & $8(14.0)$ & .21 \\
\hline AKI, N (\%) & $5(7.2)$ & $11(19.3)$ & .04 \\
\hline Wound infection, N (\%) & $4(5.8)$ & $4(7.0)$ & 1.00 \\
\hline Pneumonia, N (\%) & $14(20.3)$ & $10(17.5)$ & .70 \\
\hline
\end{tabular}

AKI, acute kidney injury; HF, heart failure; ICU, intensive care unit; LAD, left anterior descending artery; MV, mechanical ventilation

artery (PDA) to the left coronary artery (LCX) or obtuse marginal branch (OM), or from the aorta to the LCX or $\mathrm{OM}$ to the RCA or PDA. Based on related past studies, we speculated these two paths might result in different outcomes because they involve many different characteristics. For example, side-to-side anastomoses have a better patency rate than end-to-side anastomoses [Li 2014; Sewell 1974], but in SVGs, the last coronary graft usually consists of an end-to-side anastomosis. Meanwhile, studies have suggested that the physiology and lesions of the RCA are different from those of the left coronary arteries [Tinica 2019; Jeong 2013], and these different characteristics also may influence the patency rate. Therefore, this study aimed to investigate the relationship between the different paths of SVGs and surgical outcomes.

\section{MATERIAL AND METHODS}

The protocol for this retrospective study was approved by the Institutional Review Board of Chang Gung Memorial Hospital. Because the patient data were retrospective and kept anonymous, the need for signed informed consent was waived for this study.

In this retrospective study, data for 180 consecutive patients who underwent CABG between January 2011 and June 2017 were collected. Patients excluded from the study include those cases in which there was an individual vein graft, no use of left internal mammary artery (LIMA) to left anterior descending artery (LAD) bypass, the CABG was combined with other cardiac surgery, the surgery was an emergency, or there was no RCA territory (RCA and PDA) or LCX territory (LCX and OM) bypass grafting. Finally, a total of 126 patients were enrolled in this study. 


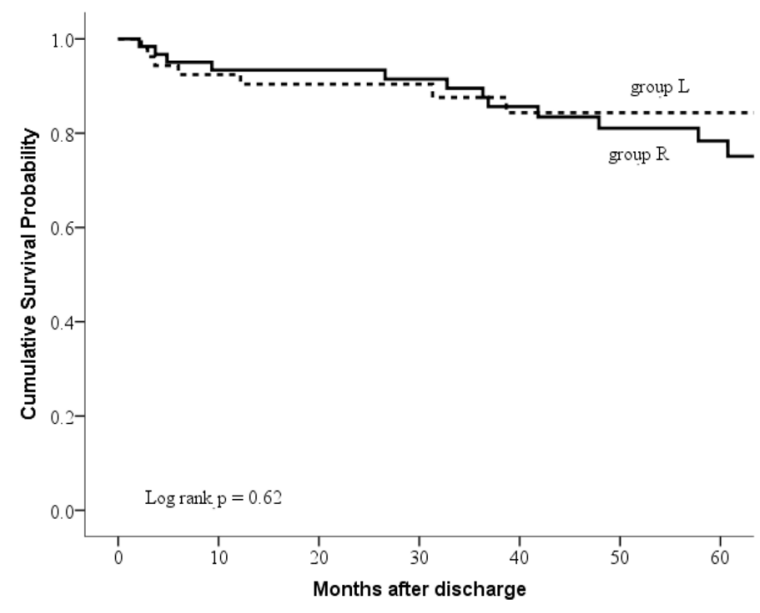

Patients at risk

\begin{tabular}{llllllll}
\hline group R & 63 & 56 & 55 & 48 & 39 & 32 & 25 \\
\hline group L & 53 & 47 & 45 & 33 & 25 & 20 & 14 \\
\hline
\end{tabular}

Figure 1. Kaplan-Meier curves of cumulative survival after survival to discharge.

The collected data included the patient's age, sex, comorbidities, preoperative clinical conditions and examination findings, operative data, surgical outcomes, and examination findings during follow-up after discharge. The RIFLE classification system was used to define acute kidney injury.

In this study, patients were divided into two groups. Specifically, if the path of the SVG was from the aorta to the RCA or PDA to the LCX or OM (that is, aorta - RCA/PDA - LCX/OM), the patients were included in Group R because, with this path, the GSV grafts generally pass along the right side of the heart lateral to the right atrium or across the atrioventricular groove. Conversely, if the path of the SVG was from the aorta to the LCX or OM to the RCA or PDA (that is, aorta - LCX/OM - RCA/PDA), the patients were included in Group L because the GSV grafts usually passed along the left side of the left ventricle.

For the patients surviving to discharge, the follow-up periods were from the surgical date until death, loss to follow-up, or the end of June 2019. The mean follow-up time was 47.45 \pm 26.84 months.

Operative method: All CABGs were performed from median sternotomy. GSVs were harvested with open methods, including the complete open technique or bridged technique. The left anterior descending artery routinely was bypassed with the LIMA unless it was not available or had poor blood flow. Each surgery was performed with the use of cardiopulmonary bypass with cardiac arrest. The cardiopulmonary bypass usually was established with aortic and right atrial cannulation. The myocardium was protected with blood cardioplegia. The sequence of anastomoses to coronary arteries was dependent on the surgeon's routine or discretion.

Statistical methods: In the description of the baseline characteristics, preoperative examination results, operative procedures, and operative outcomes, the continuous

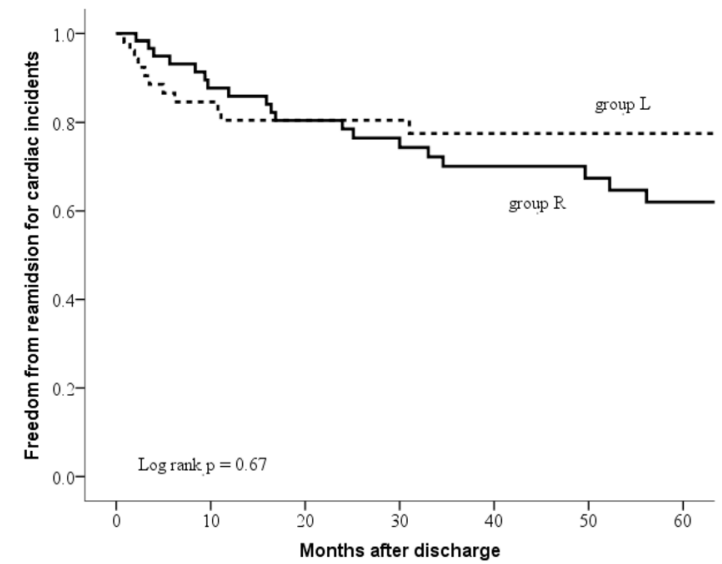

\begin{tabular}{|c|c|c|c|c|c|c|c|}
\hline group $\mathrm{R}$ & 63 & 48 & 43 & 36 & 30 & 25 & 18 \\
\hline group L & 53 & 41 & 38 & 28 & 22 & 17 & 11 \\
\hline
\end{tabular}

Figure 2. Kaplan-Meier curves of freedom from readmission for cardiac incidents after survival to discharge.

variables were presented as medians and interquartile ranges, and the categorical variables were presented as numbers and percentages.

In the univariate analysis, continuous variables were analyzed with the Mann-Whitney U test, while the Chi-square test or Fisher exact test were used to analyze categorical variables.

In the analysis comparing the rates of in-hospital mortality from heart failure and surgical complications for the two groups, the odds ratio $(\mathrm{OR})$ that was calculated from the logistic regression was used given that postoperative events usually occurred within a short time after surgery and that perioperative events, such as strokes caused by aortic manipulation, occurred during the procedure. In addition to including the EuroSCORE II predicted mortality (for in-hospital mortality from heart failure), age, sex, comorbidities, and preoperative ejection fraction (for surgical complications) into the model for adjustment, the variables that reached statistically significant differences (after verifying the correlations to avoid collinearity) were selected for adjustment.

For the patients who survived to discharge, a survival analysis was conducted using the Kaplan-Meier method and log-rank test. Similarly, when estimating the relative risk of medium-term mortality and readmission for cardiac incidents during follow-up after discharge, the hazard ratio (HR) that was calculated with the proportional hazards model was adopted. Age, sex, comorbidities, and the selected variables were included into the model for adjustment. The cardiac incidents included angina, myocardial infarction, acute heart failure with or without pulmonary edema, and atrial fibrillation.

In verifying the correlations among the variables, Spearman's rank-order correlations (with each Spearman's correlation coefficient signified by $\rho$ ) and Pearson productmoment correlations (with each Pearson's correlation coefficient signified by r) were used. 
Table 5. Odds ratios of in-hospital mortality from heart failure and acute kidney injury

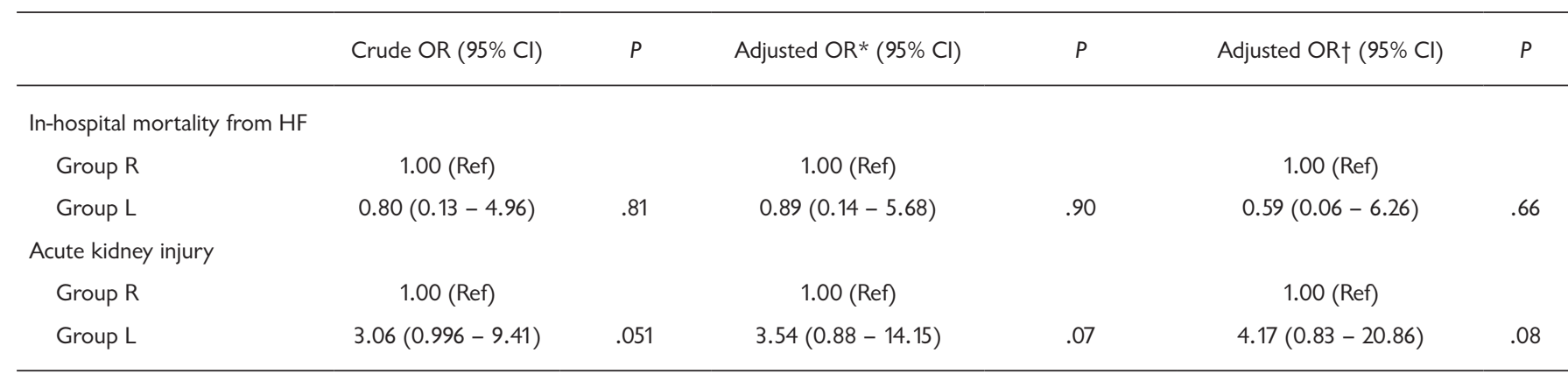

\footnotetext{
*Adjustment for age, sex, and EuroSCORE II predicted mortality for in-hospital mortality from heart failure, and age, sex, comorbidities, and preoperative ejection fraction for acute kidney injury

$\dagger$ Adjustment for $\left({ }^{*}\right)$ plus aortic cross-clamping time and number of grafts

$\mathrm{Cl}$, confidence interval; $\mathrm{HF}$, heart failure; OR, odds ratio; Ref, reference
}

Statistical analyses were conducted using SPSS for Windows (Version 17.0; SPSS, Inc., Chicago, IL, USA) and Microsoft Excel 2003.

\section{RESULTS}

There were 69 patients in Group R and 57 patients in Group L. There were no statistically significant differences between the two groups in terms of age, sex, comorbidities, or preoperative clinical conditions (Table 1). In terms of the preoperative examination findings, the ejection fractions and coronary artery luminal diameter narrowing findings of the two groups also were not significantly different (Table 2).

As shown in Table 3, Group L had a higher number of coronary artery grafts than Group $\mathrm{R}(P<.001)$, and that was correlated to a higher proportion of first diagonal branch (D1) bypass grafting $(\rho=0.63, P<.001)$ in Group L. Given that there was no D1 bypass grafting in Group R, the variable of "number of grafts" was selected for inclusion in the multivariate analysis model. Otherwise, Group L also had a shorter aortic cross-clamping time (ACT) $(P<.001)$ and total bypass time (TBT) $(P=.001)$, ACT and TBT were positively correlated with each other $(\mathrm{r}=0.769, P<.001)$, and ACT was selected for inclusion in the multivariate analysis model.

In the comparison of the post-operative outcomes (Table 4 ), it was found that the survivors in both groups had similar durations of mechanical ventilator support, intensive care unit stays, and hospital stays. However, the latter two may have been influenced by different surgeons' discretion regarding the timing of transfers to ordinary wards and discharges. Otherwise, Group L had a higher rate of acute kidney injury (AKI) $(19.3 \%$ versus $7.2 \%, P=.04)$.

Group R had 3 patients (4.3\%) who died of heart failure while still hospitalized, whereas Group L had 2 (3.5\%). The multivariate analysis results (Table 5) indicated that the SVG path was not correlated to in-hospital mortality from heart failure (crude $\mathrm{OR}=0.80, P=.81$, and adjusted $\mathrm{OR}=0.59$,
$P=$.66). Furthermore, after adjustment for age, sex, comorbidities, and the selected variables, the SVG path also was not correlated to the rate of postoperative AKI (crude OR = 3.06, $P=.051$, and adjusted $\mathrm{OR}=4.17, P=.08)$. On the other hand, preoperative creatinine clearance was an independent factor correlated with the rate of postoperative AKI (crude OR = $0.97, P=.003$, and adjusted $\mathrm{OR}=0.97, P=.04$ ).

The analysis of the medium-term survival and freedom from readmission for cardiac incidents (Figures 1 and 2) showed there were no statistically significant differences between the two groups $(\log \operatorname{rank} P=.62$ and 0.67$)$. During the follow-up after survival to discharge, in the multivariate analysis, we did not find a significant association between the SVG path and medium-term mortality and readmission for cardiac incidents before and after controlling confounding factors (Table 6). However, female gender $(\mathrm{HR}=3.40, P=.04)$, peripheral artery occlusive disease $(\mathrm{HR}=6.63, P=.01)$, history of stroke $(\mathrm{HR}=4.45, P=.02)$, and a decrease of creatinine clearance $(\mathrm{HR}=1.04, P=.002)$ were found to increase the risk of medium-term mortality. As for the risk factors of readmission for cardiac incidents, besides female gender $(\mathrm{HR}=2.83$, $P=.02)$, aortic cross-clamping time during surgery also was found to be an independent factor $(\mathrm{HR}=1.02, P=.02)$.

\section{DISCUSSION}

The outcomes and the advantages of SVG have been demonstrated by past research [An 2018; Christenson 1997; Xiao 2015; Kim 2011; Ouzounian 2010]. Similarly, the risk factors that may influence the patency of GSV grafts also have been thoroughly investigated. However, there have thus far been few studies surveying the influence of the path of coronary artery bypass grafting with SVG. Therefore, we sought in this study to determine which of the following two paths is better: aorta LCX/OM - RCA/PDA or aorta - RCA/PDA - LCX/OM.

Our results demonstrated that the path of the sequential coronary bypass grafting for Group L (aorta - LCX/OM - RCA/ 
Table 6. Hazard ratios of intermediate-term mortality and readmission for cardiac events

\begin{tabular}{|c|c|c|c|c|c|c|}
\hline \multicolumn{7}{|c|}{ Medium-term mortality } \\
\hline Group L & $0.80(0.34-1.92)$ & .62 & $1.16(0.39-3.42)$ & .80 & $1.55(0.46-5.26)$ & .49 \\
\hline \multicolumn{7}{|c|}{ Readmission for cardiac incidents } \\
\hline Group L & $0.86(0.43-1.73)$ & 0.67 & $1.10(0.52-2.33)$ & .80 & $1.99(0.80-4.93)$ & .14 \\
\hline
\end{tabular}

*Adjustment for age, sex, and comorbidities

†Adjustment for $\left(^{*}\right)$ plus aortic cross-clamping time and number of grafts

$\mathrm{Cl}$, confidence interval; $\mathrm{HR}$, hazard ratio; Ref, reference
PDA) facilitated additional D1 bypass grafting because it usually passed along the left side of heart. More specifically, $19 \%$ of the Group L patients received D1 bypass grafting, whereas none of the Group R patients did. Nonetheless, the results of this study did not indicate that $\mathrm{D} 1$ bypass grafting brought any additional benefits. It may possible to partially explain these results based on previous research which found that if the diagonal branch did not have significant stenosis, then the flow of a diagonal branch bypass graft may compete with that of a LIMA graft [Koyama 2014; Nagendran 2018]. However, in cases of unsatisfactory LIMA to LAD bypass grafting that may be due to the poor quality of the LIMA graft or hostile LAD anatomy, or to a bifurcating lesion of the $\mathrm{LAD}$ and diagonal branch, sequential bypass grafting to the diagonal branch should be a reasonable choice [Nagendran 2018; Yajima 2017].

Although the risk factors of vein graft failure are various and complex [Harskamp 2014; Harskamp 2013; Hess 2014; McKavanagh 2017], there are 3 factors that may be highly associated with SVG path. The first is the difference in anastomoses, that is, whether they are side-to-side or endto-side. Several studies have demonstrated that end-to-side anastomoses have an inferior patency rate because of frequent mismatches in the diameters of the vein grafts and coronary arteries and because of suture line intimal hyperplasia due to vessel wall stresses [Li 2014; Sewell 1974; Leuprecht 2002]. The second factor is that the use of a vein graft bypassed to the RCA territory also may have a relatively low patency rate [McKavanagh 2017]. Although there has been limited data in this regard, some research has indicated that the RCA territory has different lesions and physiology than other territories [Harskamp 2014; Harskamp 2013; Hess 2014]. The third factor is that diastolic ventricular compression may have an association with the paths of GSV grafts [Andreou 2016; Chokshi 1989; Christophides 2009]. According to several case reports, while this condition is rare, the SVG may be compressed during ventricular diastolic time, especially when heart failure occurs. Relatedly, even though those reports did not detail the paths of the vein grafts in question, it is reasonable to infer this condition might occur when the GSV graft passes lateral to the left ventricle.
As stated above, it should be reasonable to infer that SVG path from the aorta - LCX/OM - RCA/PDA should have inferior outcomes to those of the SVG path from the aorta - RCA/PDA - LCX/OM. Nonetheless, our study revealed there were no significant differences in the outcomes of the two paths. The possible explanations include, first, that most of our patients had a preoperative ejection fraction $>50 \%$ (61\% in Group R and 70\% in Group L); hence, the volume during ventricular diastole may not have severely impacted the vein graft blood flow for those patients. Second, the patients selected into this study all received LIMA to LAD grafts and vein grafts to the right and left coronary artery territories. Since these patients received almost complete revascularization, when the proximal segment had severe stenosis or occlusion, the blood flows may still have been able to partially complement one another with coronary artery anastomoses, such as the loop of Vieussens. Third, in this study, the follow-up duration may not have been long enough for the patients to accumulate severe GSV graft stenosis or occlusion. Generally speaking, although the definition of vein graft failure can be divided into early (<1 month), intermediate (or subacute) (1 month -1 year), and late (> 1 year) failure [Hess 2014], the 5 -year vein graft failure rate previously has been found to be only about $15 \%$ to $30 \%$ and the 10 -year failure rate only about $50 \%$, with those rates being found prior to the appearance of new anti-platelet or nonvitamin K antagonist oral anticoagulants to prevent thrombosis. Therefore, it is possible the medium-term mortality rate and readmission rate might have exhibited significant differences had the follow-up time been longer.

In our analysis of the surgical complications, AKI was found to be the most common surgical complication, with Group L showing a higher, but not significantly different, rate of AKI than Group R. Past research has demonstrated that the risk factors correlated to AKI after cardiac surgery are various and complex [Bove 2009; O'Neal 2016; Rosner 2006], including preoperative characteristics and clinical conditions, intraoperative techniques and the time taken to complete a cardiopulmonary bypass, and postoperative medication exposure or fluid balance control, 
among others, and data regarding most of these factors were not collected in our study or included in the multivariate analysis. Therefore, some bias may have been caused.

\section{LIMITATIONS}

In this retrospective and single-institution study, we attempted to identify the influence of the paths of vein grafts on surgical outcomes. However, there were some limitations in this study. First, the small sample size makes it difficult for the results to be generalized. Second, as mentioned above, several risk factors that may influence the blood flow of GSV grafts, such as coronary artery size, GSV size, postoperative medications, and so forth, were not included in the study. These ignored risk factors may have influenced the accuracy of our model significantly. As such, multi-institutional studies may provide further information allowing for more accurate estimations of the risks.

\section{CONCLUSION}

An SVG path from the aorta to the LCX/OM to the RCA/ PDA may facilitate extra D1 bypass grafting. However, the surgical outcomes of this path did not differ significantly from those of the other investigated path. Therefore, the demand for D1 bypass grafting may be an important consideration. At the same time, while the results for the two groups did not indicate significant influence of the SVG path, this may have been caused by the small sample size of our study. Therefore, more extensive studies would be necessary to further confirm or clarify the results of the present study.

\section{[REFERENCES}

An K, Mei J, Zhu J, Tang M. 2018. Correlates of haemodynamic flow characteristics of sequential saphenous vein grafts in coronary artery bypass grafting. Interactive cardiovascular and thoracic surgery.

Andreou AY. 2016. A case of dynamic segmental saphenous vein graft compression during diastole. Cor et Vasa 58:e444-e7.

Bove T, Monaco F, Covello RD, Zangrillo A. 2009. Acute renal failure and cardiac surgery. HSR proceedings in intensive care \& cardiovascular anesthesia 1:13-21.

Christenson, Md, PhD JT, Schmuziger, Md M. 1997. Sequential Venous Bypass Grafts: Results 10 Years Later. The Annals of thoracic surgery 63:371-6.

Christenson JT, Simonet F, Schmuziger M. 1998. Sequential vein bypass grafting: tactics and long-term results. Cardiovascular surgery (London, England) 6:389-97.

Christophides T, Georgiou G, Yiangou K. Angiographic segmental narrowing of a saphenous vein bypass graft during diastole. The Journal of invasive cardiology 2009;21:e101-2.

Chokshi SK, Meyers SN. 1989. Diastolic segmental compression of saphenous vein bypass graft. American heart journal 118:402-4.

Harskamp RE, Alexander JH, Schulte PJ, et al. 2014. Vein graft preservation solutions, patency, and outcomes after coronary artery bypass graft surgery: follow-up from the PREVENT IV randomized clinical trial. JAMA surgery 149:798-805.

Harskamp RE, Lopes RD, Baisden CE, de Winter RJ, Alexander JH. 2013. Saphenous vein graft failure after coronary artery bypass surgery: pathophysiology, management, and future directions. Annals of surgery 257:824-33.

Hess CN, Lopes RD, Gibson CM, et al. 2014. Saphenous vein graft failure after coronary artery bypass surgery: insights from PREVENT IV. Circulation 130:1445-51.

Jeong DS, Kim YH, Lee YT, et al. 2013. Revascularization for the right coronary artery territory in off-pump coronary artery bypass surgery. The Annals of thoracic surgery 96:778-85; discussion 85 .

Kim HJ, Lee TY, Kim JB, et al. 2011. The impact of sequential versus single anastomoses on flow characteristics and mid-term patency of saphenous vein grafts in coronary bypass grafting. The Journal of Thoracic and Cardiovascular Surgery 141:750-4.

Koyama S, Itatani K, Yamamoto T, et al. 2014. Optimal bypass graft design for left anterior descending and diagonal territory in multivessel coronary disease. Interactive cardiovascular and thoracic surgery 19:406-13

Leuprecht A, Perktold K, Prosi M, Berk T, Trubel W, Schima H. 2002. Numerical study of hemodynamics and wall mechanics in distal end-to-side anastomoses of bypass grafts. Journal of biomechanics $35: 225-36$.

Li H, Xie B, Gu C, et al. 2014. Distal end side-to-side anastomoses of sequential vein graft to small target coronary arteries improve intraoperative graft flow. BMC cardiovascular disorders 14:65.

McKavanagh P, Yanagawa B, Zawadowski G, Cheema A. 2017. Management and Prevention of Saphenous Vein Graft Failure: A Review. Cardiology and therapy 6:203-23.

Nagendran J, Tarola C, Catrip J, et al. 2018. Is There a Role for Diagonal Coronary Artery Stenting in Patients Undergoing Robotic Coronary Artery Bypass Graft Surgery? Journal of clinical medicine research 10:626-9.

O'Neal JB, Shaw AD, Billings FT. 2016. Acute kidney injury following cardiac surgery: current understanding and future directions. Critical care (London, England) 20:187.

Ouzounian M, Hassan A, Yip AM, et al. 2010. The impact of sequential grafting on clinical outcomes following coronary artery bypass grafting. European journal of cardio-thoracic surgery : official journal of the European Association for Cardio-thoracic Surgery 38:579-84.

Rosner MH, Okusa MD. 2006. Acute kidney injury associated with cardiac surgery. Clinical journal of the American Society of Nephrology : CJASN 1:19-32.

Sewell WH. 1974. Improved coronary vein graft patency rates with sideto-side anastomoses. The Annals of thoracic surgery 17:538-44.

Tinica G, Chistol RO, Bulgaru Iliescu D, Furnica C. 2019. Long-term graft patency after coronary artery bypass grafting: Effects of surgical technique. Experimental and therapeutic medicine 17:359-67.

Xiao F, Wang J, Wu H, Sun H. 2015. Sequential vein bypass grafting is not associated with an increase of either in-hospital or mid-term adverse events in off-pump coronary artery bypass grafting. Chinese medical journal 128:63-8.

Yajima S, Toda K, Nishi H, et al. 2017. Redo coronary bypass grafting for congenital left main coronary atresia: a case report. Journal of cardiothoracic surgery 12:26. 